\title{
Profile of Workers Tested Positive for Sars-CoV-2 in Sangalkam Health District (Senegal)
}

\author{
Bocar Baïla Diedhiou', Jean Augustin Diegane Tine², Mohamed Diabate³, Ibrahima Seck², \\ Mor Ndiaye ${ }^{4}$
}

${ }^{1}$ Dakar Medical Region, MSAS, Dakar, Senegal

${ }^{2}$ SMPSP, FMPO, UCAD, Dakar, Senegal

${ }^{3}$ University of Lyon, Lyon, France

${ }^{4}$ SOHD, FMPO-UCAD, Dakar, Senegal

Email: ^jeanaugustindiegane.tine@ucad.edu.sn

How to cite this paper: Diedhiou, B.B., Tine, J.A.D., Diabate, M., Seck, I. and Ndiaye, M. (2021) Profile of Workers Tested Positive for Sars-CoV-2 in Sangalkam Health District (Senegal). Health, 13, 1278-1284. https://doi.org/10.4236/health.2021.1311093

Received: July 29, 2021

Accepted: November 14, 2021

Published: November 17, 2021

Copyright $\odot 2021$ by author(s) and Scientific Research Publishing Inc. This work is licensed under the Creative Commons Attribution International License (CC BY 4.0).

http://creativecommons.org/licenses/by/4.0/

\begin{abstract}
Background: COVID-19 has shown the vulnerability of our healthcare staff in terms of exposure. Staff contamination is a reality to be explored and prevented. Objective: It was to describe the epidemiological, clinical, therapeutic and evolutionary profiles of health staff infected with COVID-19 in the Health District of Sangalkam (Senegal). Materials and Methodologies: This was a retrospective study from April 1, 2020 to April 20, 2021. District health staff tested positive of Sars-CoV-2 were included. Results: Sixty-six professionals were sampled, of whom seventeen were positive $(25.8 \%)$. The medical history found was diabetes type $2(n=1)$ and hypertension/obesity $(n=1)$. Only one victim had previously received an incomplete vaccination. The average age of the infected personnel was 38.4 years. Victims with an identified epidemiological link were more numerous (64.7\%). Respiratory signs (cough, rhinorrhea) and fever predominated. Caregivers accounted for $76 \%$ of the victims, compared with $26 \%$ of the administrative staff. Most patients were followed at home $(76.4 \%)$. One severe case was admitted to the intensive care unit. The recovery rate was $100 \%$. The average length of absence was 13 days. Conclusion: Infection of the staff is of great concern. Regular and decontamination and systematic cleaning of consultation offices and hospital wards are necessary. In addition, vaccination, screening, strict compliance with barrier measures (wearing masks, hand washing, physical distancing).
\end{abstract}

\section{Keywords}

Workers, Sars-CoV-2, COVID-19, Health-District, Senegal 


\section{Introduction}

The 1st cases of infection by the new respiratory-tropic coronavirus, currently named Sars-CoV-2, were identified in December 2019 [1]. The meteoric propensity of COVID-19 has had a devastating impact on the economic and psychological well-being of people. Drastic measures such as quarantine, physical distancing or massive restriction of public life to avoid the collapse of the health system have been implemented in Senegal. The clinical manifestations associated with COVID-19 appear to be highly polymorphic and multi-systemic, going far beyond the typically respiratory nosologic framework. These manifestations can be hematological, cardiovascular, dermatological, hepatic, renal, digestive, ophthalmological and even neurological [2]. This virus rapidly became international in a few months, taking on the appearance of a pandemic considered to be the largest global crisis since the Second World War. In Africa, after Algeria, Egypt and Nigeria, Senegal confirmed its first case on March 2nd, 2020, a French national who returned to Dakar on February 26th, 2020. In the Sangalkam Health District, the first positive cases were reported on March 25th, 2020. They were five imported cases from the United States. The contamination will progressively spread in the district population and will affect the health staff who will record their first case on June 8,2020 . The objective of this study was to describe the epidemiological, clinical, therapeutic and evolutionary profiles of COVID-19 in the staff of the Sangalkam Health District. The aim was to identify the determinants of staff contamination in order to develop protection strategies.

\section{Materials and Methodologies}

Field of study: The Sangalkam Health District was created by decree No. 031575/MSAS/SG/BL of 31/12/2019. It was created by splitting the Rufisque Health District and is composed of 14 health posts (HPs) and a reference health center. Its surface area is $195 \mathrm{~km}^{2}$, which is one third of the Dakar Region, the administrative capital of Senegal. Its population is estimated at 175,000 inhabitants in 2021. It polarizes four municipalities: Sangalkam, Bambilor, Jaaxay and Tivaoune Peul. In Senegal, the health district is the base of the health pyramid and represents a geographic, operational and administrative entity that provides health services and care to the population. It is the most decentralized or peripheral level of the health system.

Type and period of study: this is a retrospective study from April 1, 2020 to April 20, 2021 which focused on health establishments in Sangalkam District to find the agents who were victims of COVID-19.

Enrollment of COVID-19 cases: The suspicion of COVID-19 was made in the following situations:

- Presence of influenza-like illness (sudden onset fever associated with at least two of the following symptoms: nasal congestion, pharyngitis, cough, myalgias, arthralgias, headache, asthenia, maculo-papular rash);

- The occurrence of acute respiratory distress syndrome (ARDS); 
- Recent contact with a confirmed COVID-19 subject.

All suspects' cases had a nasopharyngeal swab taken and sent to the Pasteur Institute in Dakar. Suspects who were tested positive with Reverse Transcriptase-Polymerase Chain Reaction (RT-PCR) for SARS-CoV-2 were included in this study.

Data collection: to carry out this study, we were interested in the investigation forms, the analysis results bulletins of the Pasteur Institute of Dakar, and the medical records of the patients. Parameters such as socio-professional characteristics, circumstances of contamination, clinical, para-clinical, therapeutic and evolutionary data were exploited.

Data analysis: the data were analyzed by Epi-info.

Ethical aspects: the administrative health authorities of this Health District gave their authorization for the realization of this work. The confidentiality of the collected data was guaranteed.

\section{Results}

Sixty-six healthcare workers meeting the definition of a suspect case were sampled. Seventeen (25.8\%) were positive for COVID-19. The majority of victims, $88.2 \%(\mathrm{n}=15)$, were female. Males represented $11.8 \%(\mathrm{n}=2)$. The average age of the victims was 38.4 years. The ages ranged from 24 to 69 years. Two of the victims were pregnant at the time of screening. One patient had type 2 diabetes and one had hypertension/obesity with a BMI of 35.2. Community health workers represented $35.3 \%(n=6)$ of the victims. The distribution of COVID-19 positive cases by occupation shows a variety of types of workers infected (see Table 1).

During the 1st wave (March 2020 to November 31, 2020), infected professionals represented $53 \%(n=9)$ of the victims. In the 2 nd wave (December to April $20,2021)$, infected staff represented $47 \%(\mathrm{n}=8)$. The Sangalkam health center recorded the most workers positive for the COVID-19 test (see Table 2).

The clinical picture was polymorphic. Rhinorrhea, fever and cough were more frequent (see Figure 1). The type of specimen taken was nasopharyngeal. The

Table 1. Distribution of positive COVID-19 cases by profession.

\begin{tabular}{ccc}
\hline Profession & $\begin{array}{c}\text { Absolute frequency } \\
\text { (n) }\end{array}$ & $\begin{array}{c}\text { Relative frequency } \\
(\%)\end{array}$ \\
\hline Community health worker & 6 & 35.3 \\
Midwife & 3 & 17.6 \\
Senior Nurse & 3 & 17.6 \\
Hygiene and cleaning technician & 2 & 11.8 \\
Social worker & 2 & 11.8 \\
Driver & 1 & 5.9 \\
Total & 17 & 100 \\
\hline
\end{tabular}




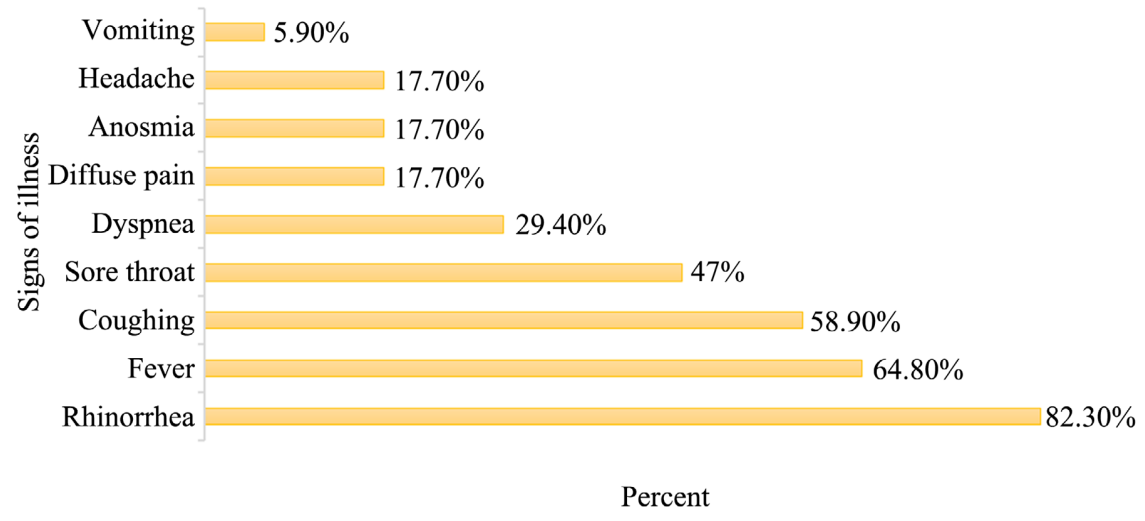

Figure 1. Distribution of signs of COVID-19 among victims $(n=17)$.

Table 2. Distribution of positive COVID-19 cases by facility.

\begin{tabular}{ccc}
\hline Facility & Absolute frequency (n) & Relative frequency (\%) \\
\hline Sangalkam Health Center & 11 & 64.6 \\
Bambilor Health Post & 2 & 11.8 \\
Kounoune Health Post & 1 & 5.9 \\
Apix Health Post & 1 & 5.9 \\
Niakoulrab Health Post & 1 & 5.9 \\
Tivaoune Peul Health Post & 1 & 5.9 \\
Total & 17 & 100 \\
\hline
\end{tabular}

diagnosis was made by PCR. Receipt of the result varied from 2 to 3 days. The majority of cases, $94.1 \%(\mathrm{n}=16)$, were simple or moderate. One severe case (5.9\%) with respiratory distress was observed. In relation to the place of follow-up, $17.7 \%(\mathrm{n}=3)$ were followed up at the level of the epidemiological treatment centers (ETC), 5.9\% $(\mathrm{n}=1)$ were transferred to intensive care and $76.4 \%(n=13)$ were followed up at home. Treatment was symptomatic, based on azithromycin. Vitamin C, corticosteroid therapy, anti-influenza drugs, oxygen therapy $(\mathrm{n}=1)$. No deaths were recorded. Cure was announced on the basis of clinical (disappearance of symptoms) and biological (2 separate 48-hour control negativities) arguments. The number of days of sick leave varied from 8 to 27 days, with an average of 13 days of absence.

\section{Limit of Study}

The limit of this study is the lack of biological and radiological data. The sample size is small so we did not do statistical tests.

\section{Discussion}

The health districts in Senegal have played a major role in the fight against COVID-19. They have actively contributed to the detection of suspected cases, 
the monitoring of positive cases and the follow-up of contacts. In addition, they promoted communication and health education activities and transmitted daily health data to the Ministry of Health and Social Action (MSAS) on the evolution of the pandemic. This primary place occupied by the districts has contributed exponentially to the exposure of health personnel who are the front line in the fight against HIV/AIDS. In the Sangalkam Health District, a quarter of the staff tested were positive for COVID-19 during the study period. In his study, Lebreton observed a higher rate of contamination of the staff (30\%). Moreover, he observed an average age similar to ours (38 years) and a predominance of women [3]. As a matter of fact, the vast majority of the staff, especially nursing staff, are women in Sangalkam Health District. Studies have also confirmed this trend towards feminization of the medical and paramedical profession [4]. Several factors explain this rather high rate of infected personnel. One factor is the obligation for health care workers to systematically take charge of all patients, regardless of their clinical condition. The lack of protective equipment is a factor that increases the risk. In addition, failure to strictly adhere to recommended barrier measures is also a contributing factor. The staff of the health center and the administrative block were the most affected by the pandemic, cumulating $64.6 \%$ of positive staff. Indeed, the administrative block is located outside the referral health center, at a distance of about 150 meters. The proximity between the two increases the back and forth movement and facilitates the spread of the infection on both sides. Other geographical factors contributed to these results. These included the COVID samples taken in the administrative block, which houses the COVID-19 sampling room for the entire District. In addition, patients with suspicious signs were isolated at the Health Center in a dedicated isolation room. Severe cases of COVID-19 in the district awaiting transfer to the reanimation services were temporarily managed at the Health Center. On the individual level, certain prohibited acts carried out by the personnel increased the risk. These included the sharing of tools and communal meals. Indeed, the Center was confronted with a real lack of space. For example, the on-duty room was shared by both the maternity and medical staff. In relation to the health pyramid, referrals of suspected cases from the health posts (HP) to the Health Center propel the risk of contamination of the Health Center staff. At the level of the Administrative Block, the lack of premises facilitates the spread of contamination. Thus, three of the administrative agents were grouped together in a promiscuous office of about $12 \mathrm{~m}^{2}$, making it difficult to maintain physical distance, and all tested positive during the same week. This vulnerability was exacerbated by the hugging and communal sharing of meals practiced by these staff. In addition, the many district activities carried out in the administrative block, such as the district coordination meetings to which all district head nurses and midwives are invited, encourage grouping. During these meetings, the physical distance was almost not respected. All of these observations support our findings. Thus, seven of the infected staff had a correlable epidemiological link with colleagues who were victims of COVID-19. These observations confirm the in- 
terprofessional contamination, hence the need to interrupt the chain of contamination by screening. The proportion of community cases was also significant (35.3\%). Indeed, community transmission occurs when a person contracts COVID19 from an unknown source. In our study, secondary cases were predominantly correlated with positive caregivers. Several factors favoring contamination were identified. These included the absence of a hygienist for regular decontamination of consultation offices, hospital wards and waiting rooms. The observations also showed that the staff strictly respected the protection measures in case of a suspicious clinical picture or a positive patient. However, studies have shown that the proportion of asymptomatic forms is still debated and is probably around $15 \%$ [5]. Human-to-human transmission of Sars-CoV-2 is known, with a basic reproduction rate of between 2 and 4 [6]. This means that an infected individual infects on average 2 to 4 other individuals. Our results are in the same direction. Indeed, during the 2nd wave, 3 of the 4 positive cases in the administrative block shared the same office and were all diagnosed positive during the same week. The 4th case tested the following week was the driver of this district management team. Studies have shown that the predominant mode of transmission would involve large droplets $(>5 \mu \mathrm{m})$ generated during speech, coughing, or sneezing and not spreading more than two meters. There is strong evidence for airborne transmission [7], mediated by aerosols (droplets $\leq 5 \mu \mathrm{m}$ in size), but this route is marginal, as is transmission by contact with contaminated surfaces. The classic manifestations of the disease are those of a respiratory virosis, ranging from mild upper respiratory involvement to severe pneumonia. The symptoms most frequently reported in the literature are cough, fever and dyspnea [8]. The analysis of our results shows a polymorphism of the picture with a predominance of respiratory signs (rhinorrhea, cough, and dyspnea) and general signs (fever). The contamination of caregivers increases their psychosis, their demotivation and also the risk of contamination of the environment (family and inter-professional) and of the people using the health structures. In our study, no case of death was recorded. In fact, the clinical pictures were not very serious, probably due to the low average age. In France, the mortality associated with COVID-19 is estimated at $0.7 \%$, with wide variations according to the terrain [9]. Age over 65 years is the main risk factor for severe disease. The rapid spread of the virus explains the major impact on the health care system with the sudden saturation of care structures for COVID-19 patients. Thus, most of the health professionals affected by COVID-19 were followed up at home. However, it is difficult to ensure that these patients comply with home confinement. In addition, some victims lived in overcrowded homes where compliance with physical distancing was almost impossible. This increases the psychosis of those around them.

\section{Conclusion}

Staff contamination represents a major concern. It generates a major demotivation among the personnel, favors absenteeism and constitutes an obstacle for the 
continuity of care. Thus, the sensitization and the protection of this personnel are essential. The availability of protective materials (masks, gloves) and the strict respect of barrier gestures are essential. These include regular hand washing with soap and water and/or the use of hydro-alcoholic solution; the prohibition of handshakes and hugs; and the respect of physical distance $(1.5 \mathrm{~m})$. In addition, regular, systematic decontamination of hospital wards and the construction of new, more suitable premises are essential. In addition, vaccination and massive screening of personnel are essential levers to fight against the contamination of personnel.

\section{Conflicts of Interest}

The authors declare that they have no personal interest.

\section{References}

[1] Zhu, N., Zhang, D.Y., Wang, W.L., Li, X.W., Yang, B., Song, J.D., et al. (2020) A Novel Coronavirus from Patients with Pneumonia in China, 2019. The New England Journal of Medicine, 382, 727-733. https://doi.org/10.1056/NEJMoa2001017

[2] Kashongwe, I.M., Lepira, F.B., Situakibanza, H.N.T., Makulo, J.R.R., Sumaili, E.K. and Kayembe, J.M.N. (2020) Clinical Manifestations of SARS-Cov-2 (COVID-19) Coronavirus Infection. Annals of African Medicine, 13, 3685-3693.

[3] Lebreton, C., Brunel, A., Bouiller, K. and Chirouze, C. (2020) Risk Factors for COVID-19 in Caregivers. Médecine et Maladies Infectieuses, 50, S68. https://doi.org/10.1016/j.medmal.2020.06.132

[4] Diédhiou, B.B., Ba, E.H.M., Coly, O., Ba, F., Tine, J.A.D. and Sankharé, B. (2019) Psychological, Physiological, Somatic and Socio-Professional Impacts of Night Work among Staff at the Regional Hospital of Kolda (Senegal). IJIAS, 26, 582-588.

[5] Byambasuren, O., Cardona, M., Bell, K., Clark, J., McLaws, M. and Glasziou, P. (2020) Estimating the Extent of Asymptomatic COVID-19 and Its Potential for Community Transmission: Systematic Review and Meta-Analysis. JAMMI, 5, 223 234. https://doi.org/10.3138/jammi-2020-0030

[6] Liu, Y., Gayle, A.A., Wilder-Smith, A. and Rocklöv, J. (2020) The Reproductive Number of COVID-19 Is Higher Compared to SARS Coronavirus. Journal of Travel Medicine, 27, taaa021. https://doi.org/10.1093/jtm/taaa021

[7] Gehanno, J.F., Bonneterre, V. and Andujar, P. (2020) Arguments for a Possible Airborne Transmission of Sars-CoV-2 in the COVID-19 Crisis. Archives des Maladies Professionnelles et de P Environnement, 81, 306-315. https://doi.org/10.1016/j.admp.2020.04.018

[8] Pascarella, G., Strumia, A. and Piliego, C. (2020) COVID-19 Diagnosis and Management: A Comprehensive Review. Journal of Internal Medicine, 288, 192-206. https://doi.org/10.1111/joim.13091

[9] Ministry of Health in Senegal (2020) COVID-19: Epidemiological Report of July 9, 2020. National Epidemiological Bulletin. 